\title{
Neue Machtbalance im Lokalpräsidentialismus? Vom Scheitern der kommunalen Direktdemokratie in Frank- reich
}

\author{
Sabine Kuhlmann/Philipp Richter
}

\section{A. Einleitung}

Ausgehend von einer überwiegend repräsentativen Ausgestaltung der lokalen Demokratie ist es in vielen Ländern zu Vorstößen in Richtung verstärkter direktdemokratischer Teilhabe gekommen. ${ }^{1}$ Auch in Frankreich durchlief das traditionell repräsentativ-demokratisch geprägte lokale Politikmodell in den vergangenen zwei Jahrzehnten eine Erneuerung, deren Ausmaß und Reichweite im Folgenden untersucht werden soll. Es wird gezeigt, dass, unbeschadet der inzwischen vorhandenen direktdemokratischen Instrumentarien auf lokaler Ebene, die bindenden lokalen Referenden die überkommene, auf den Bürgermeister zugeschnittene Politikkultur nicht grundlegend verändert haben. Vielmehr kam es zur Inbesitznahme der neuen direktdemokratischen Beteiligungsinstrumente durch die lokalen Exekutiven, deren Stellung im Ergebnis sogar noch bekräftigt wurde.

\section{B. Das traditionelle System lokaler Demokratie in Frankreich}

Frankreich gilt in der vergleichenden Lokalforschung als ein ,politisch starkes“ Kommunalmodell. $^{2}$ Die Gemeinden sind nicht nur Selbstverwaltungsinstanzen, sondern auch Arenen politischer Willensbildung und haben - analog zur nationalen Ebene - eigene lokale Politiksysteme ausgebildet. Die vergleichsweise hohe und relativ stabile Beteiligung an Kommunalwahlen, die seit der Nachkriegszeit bei durchschnittlich zwischen $60 \%$ und $70 \%$ liegt und damit wesentlich höher ist als in anderen Ländern, wie etwa Großbritannien oder den USA, darf als Indiz für den hohen politisch-demokratischen Rang, den die lokale Ebene in der Poli-

1 Vgl. United Cities and Local Governments, Decentralization and local democracy. First Global Report, Barcelona 2008, S. 156.

2 Vgl. S. Kuhlmann, Politik- und Verwaltungsreform in Kontinentaleuropa. Subnationaler Institutionenwandel im deutsch-französischen Vergleich, 2009, S. 62 ff. 
tikkultur Frankreichs einnimmt, angesehen werden. Auch wenn die Wahl den Mitgliedern der Gemeinderäte gilt und das französische Kommunalmodell somit formell am parlamentarischen System orientiert ist, hat sich in der Handlungsrealität jedoch seit langem das Modell eines „,städtischen Präsidentialismus“3 ${ }^{\text {3 }}$ herausgebildet, für den eine Dominanz, wenn nicht „Allmacht“ der Exekutive und eine schwache Stellung des Rates und der Parteien charakteristisch sind; allerdings mit Varianzen zwischen den (wenigen) Großstädten und den (zahlreichen) Kleinkommunen. Der französische Bürgermeister profitiert dabei einerseits von seinem historisch gewachsenen Prestige eines Notabeln, was sich bis in die 1980er Jahre hinein in seiner bemerkenswert langen politischen Lebensdauer und in dem Phänomen der ,politischen Vererbung“ zeigt. ${ }^{4}$ Seine indirekte Wahl kommt dadurch, dass die potenziellen Kandidaten für das Bürgermeisteramt praktisch immer den ersten Listenplatz einnehmen und infolge des Listenwahlsystems, in dem keine Panaschierung und Änderung der Reihenfolge der Kandidaten vorgesehen sind, zudem faktisch einer Direktwahl gleich. ${ }^{5}$ Andererseits trägt zur herausragenden Stellung des französischen Bürgermeisters die im europäischen Kontext einmalige Möglichkeit der Ämterhäufung (cumul des mandats) bei, die es den lokalen Amtsträgern erlaubt, Mandate der verschiedenen Politikund Verwaltungsebenen zu kumulieren und dadurch ihre politischen Einflusssphären weit über ihren Wahlkreis hinaus bis auf die nationale Politikebene auszudehnen.

Ungeachtet dieser Verfassungsrealitäten ist die Ausgestaltung der lokalen Demokratie in Frankreich vom Grundzug der repräsentativen Demokratie bestimmt. Direkte Teilhaberechte waren auf lokaler Ebene traditionell wenig ausgeprägt. Das (konsultative) Referendum, welches von den französischen Bürgermeistern bereits im 19. Jahrhundert, allerdings ohne rechtliche Grundlage, angewendet worden war, wurde erst im Jahre 1971 rechtlich kodifiziert. ${ }^{6}$ Jedoch blieb es auf Konsultationen über Gemeindezusammenschlüsse beschränkt. Sämtliche gesetzgeberischen Versuche, direktdemokratische Strukturen rechtlich ver-

3 A. Mabileau, Kommunalpolitik und -verwaltung in Frankreich: das „lokale System“ Frankreichs, 1996, S. 83.

4 Vgl. V. Hoffmann-Martinot, Reform and Modernization of Urban Government in France, in: V. Hoffmann-Martinot/H. Wollmann (Hrsg.), State and Local Government Reforms in France and Germany. Divergence and Convergence, 2006, S. 231 (239 f.).

5 Vgl. Mabileau, Kommunalpolitik (Fn. 3), S. 65.

6 Vgl. M. Paoletti, La pratique politique du référendum local: une exception banalisée, in: Centre de recherches administratives, politiques et sociales [CRAPS]/Centre universitaire de recherches administratives et politiques de Picardie [CURAPP] (Hrsg.), La démocratie locale. Représentation, participation et espace public, Paris 1999, S. 219 (221). 
bindlich einzuführen, scheiterten am Widerstand der im Parlament und im Senat vertretenen Bürgermeister, die um ihre Vormachtstellung im lokalen Raum fürchteten und ihren Einfluss auch über die Interessenverbände, vor allem die Association des Maires de France (A.M.F.), geltend machten. Bis in die 1990er Jahre blieb die Debatte um lokale Referenden selbst innerhalb der neuaufkommenden Diskussionen um ,partizipative Demokratie“ vollkommen randständig, wenn nicht gänzlich bedeutungslos.

\section{Reformen lokaler Demokratie}

\section{Konsultativreferenden zu Beginn der 1990er Jahre}

Erst mit dem Gesetz von 1992 fanden lokale Referenden - jenseits von Gemeindezusammenschlüssen als bis dahin einzig zulässigem Abstimmungsthema Eingang in die französische Gesetzgebung. ${ }^{7}$ Zugleich wurden aber auch ihr ausschließlich konsultativer Charakter (consulations des électeurs) und eine Vielzahl von Verfahrenshürden und -beschränkungen festgeschrieben. Besonders hervorzuheben sind der explizite Ausschluss des Bürgerbegehrens und die Regelung, dass Referenden nur vom Bürgermeister oder auf schriftlichen Antrag von mindestens der Hälfte der Ratsmitglieder (in Kommunen unter 3.500 Einwohner) bzw. von einem Drittel der Ratsmitglieder (in Kommunen über 3.500 Einwohner) ausgelöst werden konnten. Darüber hinaus enthält das Gesetz zahlreiche rechtliche Vorgaben zur Durchführung der Abstimmung, zum zeitlichen Rahmen, zum Ablauf und zu den Bedingungen, unter denen Referenden unzulässig sind. ${ }^{8}$

Beschränkten sich kommunale Referenden zunächst auf den räumlichen Bereich der Einzelkommune, konnten mit dem Gesetz des Jahres 1995 auch die interkommunalen Gemeinschaften (EPCI - établissements publics à coopération intercommunale) von der Möglichkeit Gebrauch machen, die Bürger ihrer Mitgliedskommunen, allerdings ausschließlich in Fragen der Raumordnung (aménagement), durch Referenden zu konsultieren. ${ }^{9}$ Zudem kann seit dem Gesetz von 1995 ein lokales Referendum auch durch ein Fünftel der Wählerschaft einer Kommune bzw. einer EPCI, allerdings ebenfalls nur für raumbezogene

7 Loi no. 92-125 du 6 février 1992 relative à l'administration territoriale de la République.

8 Vgl. Paoletti, Pratique politique (Fn. 6), S. 233.

9 Loi no. 95-115 du 4 février 1995 d'orientation pour l'aménagement et le développement du territoire. 
Maßnahmen und nach wie vor ausschließlich konsultativ, initiiert werden. Außerdem wurden wiederum zahlreiche Vorkehrungen getroffen, um die Anwendung und Wirksamkeit von Referenden möglichst zu begrenzen. So wurde festgeschrieben, dass lokale Referenden mit Beginn des Jahres, welches dem Jahr der nächsten Kommunalwahl vorausgeht, ebenso untersagt sind wie während der Wahlkampfzeit (direkte wie auch indirekte Wahlen). Außerdem musste zwischen zwei Referenden, die den gleichen Gegenstandsbereich betreffen, ein zeitlicher Abstand von mindestens zwei Jahren und generell zwischen zwei Referenden ein Abstand von einem Jahr eingehalten werden.

In der Gesetzgebung zum lokalen Referendum, die erst nach mehrmaligen Anläufen und dann auch nur zaghaft in Gang gekommen war, spiegelt sich deutlich das Festhalten am repräsentativ-demokratischen und „exekutiv-lastigen“ lokalen Politikmodell wider. Der erfolgreiche Widerstand gegen einen Ausbau und eine erweiterte Bindungswirkung von lokalen Beteiligungsverfahren erklärt sich wiederum aus der Schlüsselstellung der Bürgermeister, die als Symbolfigur und „Inkarnation“ des repräsentativ-demokratischen Politikmodells ihren Einfluss auf den nationalen Gesetzgebungsprozess geltend machten. ${ }^{10}$ So wurde die rechtliche Verankerung des lokalen Referendums von den BürgermeisterAbgeordneten und Bürgermeister-Senatoren als uniformierender jakobinischzentralistischer Eingriff in „lokale Angelegenheiten“ kritisiert und eine etwaige Einführung bindender Referenden verhindert. ${ }^{11}$

\section{Bindende Referenden seit dem Jahr 2003}

In jüngerer Zeit ist im Zusammenhang mit der zweiten Dezentralisierungswelle (sog. Acte II) erneut Bewegung in die Debatte um direktdemokratische Teilhabe auf lokaler Ebene gekommen. ${ }^{12}$ Die Demokratisierung der lokalen Ebene und der Ausbau der direkten Demokratie werden als Bestandteile einer umfassenden Modernisierung von Staat und Politik in Frankreich und als Erfolgsfaktoren der zweiten Dezentralisierungsetappe gesehen. ${ }^{13}$ Zum einen ist das lokale Referen-

10 Vgl. R. Matuszewicz, Représentations et Pratiques des conseils de quartier: une démocratie participative en devenir sous contrainte, in: S. Guérard (Hrsg.), Crise et mutation de la démocratie locale. En Angleterre, en France et en Allemagne, Paris 2004, S. 199 (202).

11 Vgl. Paoletti, pratique politique (Fn. 5), S. 220.

12 Vgl. S. Kuhlmann, Reforming local public services: trend and effects in Germany and France, in: Public Management Review 2008, 573 (574 ff.).

13 Vgl. F. Rangéon, Le levier institutionnel: référendum et réforme électorale, in: G. Marcou/H. Wollmann (Hrsg.), Annuaire 2004 des collectivités locales, Paris 2004, S. 53. 
dum mit der Verfassungsänderung vom März 2003 in Art. 72-1 der französischen Verfassung aufgenommen worden und hat damit nun Verfassungsrang. Sein Anwendungsbereich wurde auf alle Gebietskörperschaften, d.h. auch auf die Departements und Regionen, ausgeweitet, was aber zugleich bedeutet, dass die EPCI ausdrücklich ausgeschlossen sind. Zum anderen wurde mit der Einführung von bindenden lokalen Referenden (référendum décisionnel) durch das $\mathrm{Ge}-$ setz vom 1.8.2003 ${ }^{14}$ eine Bresche in das bisherige repräsentativ-demokratische Modell geschlagen. ${ }^{15}$ Allerdings ist der französische Gesetzgeber nicht soweit gegangen, für die Initiierung des Referendums ein Bürgerbegehren vorzusehen, sondern beschränkte sich auf das Initiativrecht des Rates, der seinerseits auf Vorschlag des Bürgermeisters bzw. Ratspräsidenten (Region/Departement) darüber abstimmt, ob ein Sachverhalt zum Gegenstand eines Referendums wird. ${ }^{16}$ Damit ein Referendum erfolgreich ist, muss zudem mindestens die Hälfte der Wähler an der Abstimmung teilgenommen und von dieser die Mehrheit zugestimmt haben (Art. L.O. 1112-7), was auch als prohibitiv wirkende Hürde bezeichnet worden ist. ${ }^{17}$ Es können alle Sachverhalte, die im Kompetenzbereich der jeweiligen Gebietskörperschaft stehen, Gegenstand eines lokalen Referendums werden (Art. L.L. 1112-1). Lediglich individuelle Verwaltungsakte, wie z.B. die Ausstellung einer Baugenehmigung, sind hiervon ausdrücklich ausgeschlossen (Art. L.O. 1112-2). Insgesamt ist darin, dass im Falle der Durchführung eines Referendums dessen Abstimmungsergebnis dann bindend ist, sowie darin, dass diese Regelung für alle Gebietskörperschaften gilt und keine inhaltliche Beschränkung enthält, ein bemerkenswerter Vorstoß in Richtung verstärkter direktdemokratischer Teilhabe in französischen Kommunen zu erblicken.

14 Loi organique no. 2003-705 du 1er août 2003.

15 Mit der Einführung des bindenden lokalen Referendums sind einerseits die „alten“ Regelungen zum Konsultativreferendum grundsätzlich außer Kraft getreten. Andererseits wurde die im Gesetz von 1995 festgeschriebene Konsultation der Wähler zu Fragen der Stadtentwicklung (aménagement), bei denen auch das Bürgerbegehren vorgesehen ist, beibehalten (Art. L. 5211-49 CGCT). Vgl. G. Marcou, Décentralisation: Quelle théorie de l'Etat? in: G. Marcou/H. Wollmann (Hrsg.), Réforme de la décentralisation, réforme de l'Etat, régions et villes en Europe. Annuaire 2004 des collectivités locales, Paris 2004, S. 235 (246).

16 Die französische Verfassung sieht neben dem lokalen Referendum auf Initiative der lokalen Gebietskörperschaft auch die Variante vor, dass lokale Referenden durch die Zentralregierung oder vom Präsidenten der Republik initiiert werden können. Vgl. Rangéon, Le levier institutionnel (Fn. 13), S. 55.

17 Vgl. C. Prémat, The growing use of referenda in local politics: a comparison of France and Germany, in: H. Reynert u.a. (Hrsg.), Revolution or renovation? Reforming local Politics in Europe, Brügge 2005, S. 185 (187). 


\section{Reformwirkungen}

\section{Anwendungshäufigkeit lokaler Referenden}

Zur Anwendungspraxis von lokalen Referenden liegen Erkenntnisse vor allem für das Konsultativreferendum vor, wohingegen es für bindende lokale Referenden seit ihrer Einführung im Jahre 2003 bis zum Jahre 2007 kein einziges Beispiel gab. Die Nichtanwendung wurde einerseits auf die erwähnten prohibitiv wirkenden Verfahrenshürden (Beteiligungsschwelle von 50\%) zurückgeführt. ${ }^{18}$ Andererseits ist sogleich einzuwenden, dass diese Schwelle bei konsultativen Referenden in Frankreich durchaus überwunden wird, da dort die durchschnittliche Beteiligungsquote zwischen 1995 und 2004 bei 55,5\% $(n=125)$ lag. ${ }^{19}$ Damit erklärt sich die „Anwendungslücke“ vor allem daraus, dass die repräsentativen Gremien davor zurückschrecken, eine Abstimmung zu initiieren, deren Ergebnis negativ für sie ausgehen könnte und dennoch bindend wäre. Aber auch Konsultativreferenden, auf die sich die Analyse daher im Folgenden beschränken muss, kommen vergleichsweise selten vor. Besonders rar sind Referenden durch Bürgerinitiativen (initiative populaire), von denen bisher nur insgesamt neun Fälle registriert wurden, bei denen die $20 \%$-Hürde bewältigt worden ist. Diese neun Popularinitiativen waren mehrheitlich erfolglos, sei es, dass Themen unzulässig waren (Kommune Azé), sei es, dass der Rat es trotz formaler Gültigkeit ablehnte, die Abstimmung durchzuführen (Kommunen Mourenx, Geispolsheim, Lancieux, Baudreix). Insgesamt ist das Bürgerbegehren damit in der lokalen Anwendungspraxis zu vernachlässigen.

„Von wenigen Ausnahmefällen abgesehen, sieht sich der Bürgermeister nie der Forderung nach einem Referendum von Seiten der Bürger durch Bürgerbegehren gegenüber. “20

Bei den ratsinitiierten Konsultativreferenden (consultations) ist insgesamt eine etwas größere Häufigkeit als bei Bürgerinitiativen festzustellen. So hatte die rechtliche Kodifizierung von lokalen Referenden über Gemeindezusammenschlüsse im Jahre 1971 sogleich einige französische Bürgermeister ermutigt, dieses Instrument anzuwenden, und zwischen 1971 und 1993 wurden insgesamt 202 kommunale Referenden durchgeführt. Seit der Gesetzgebung des Jahres 1995 bis

18 Vgl. Prémat, Use of referenda (Fn. 17), S. 187.

19 Die Beteiligungsquote ist dabei in Kommunen mit unter 10.000 Einwohnern durchschnittlich höher $(60,2 \% ; n=227)$ als in Kommunen mit über 10.000 Einwohnern $(41 \%$; n=31). Vgl. F. Fritz, Lokale Referenden in Deutschland und Frankreich - ein empirischer Vergleich. Unveröff. Diplomarbeit Konstanz, 2006, S. $71 \mathrm{ff}$.

Paoletti, Pratique politique (Fn. 6), S. 228 (Übersetzung S.K.). 
2004 gab es immerhin weitere 184 Ratsinitiativen. Insgesamt ist dennoch auch hier die Anwendungspraxis auffallend gering. Die durchschnittliche Häufigkeit zwischen 1971 und 1993 lag bei nur 8 Referenden pro Jahr und noch nicht einmal 0,01 pro Kommune. ${ }^{21}$ Im Zeitraum 1995 bis 2004 waren es dann immerhin landesweit durchschnittlich 18 pro Jahr. Die geringe Häufigkeit lässt sich auch daran ablesen, dass es seit 1995 in ganz Frankreich nur eine Kommune gibt, in der mehr als eine Abstimmung stattfand (Couilly-Pont-aux-Dames mit 1908 Einwohnern). Hinzu kommt, dass ein erheblicher Anteil der Ratsinitiativen (insgesamt 20\%) von der Aufsichtsbehörde für unzulässig erklärt wurde, vor allem, weil der Abstimmungssachverhalt nicht in den Kompetenzbereich der Kommune fiel.

\section{Stärkung der Macht der Mehrheit und der Exekutive}

In der Frage, inwieweit die Anwendung der neuen direktdemokratischen Instrumente das Gesamtsystem lokaler Politik in Frankreich verändert hat und sich ein systemtransformierender Wandel vom ursprünglich repräsentativ-demokratischen, exekutiv dominierten und Macht konzentrierenden Modell hin zu einem direktdemokratisch ,angereicherten“ Politiksystem mit machtbeschränkenden Gegengewichten für die Exekutive vollzogen hat, ergibt sich ein ausgesprochen negatives Bild. Abgesehen von der fast zu vernachlässigenden Anwendungshäufigkeit des Bürgerbegehrens und dem vollständigen Verzicht auf die Anwendung des bindenden lokalen Referendums, hält sich auch die Wirkung des Referendums als Handlungsoption in Grenzen. Die Behauptung, mit der rechtlichen Kodifizierung lokaler Referenden werde das Ende der repräsentativen Demokratie und des französischen „Bürgermeisters“ als dessen Inkarnation eingeläutet, geht an der lokalen politischen Realität vollkommen vorbei. Denn die bisherige Praxis direktdemokratischer Beteiligung hat in erster Linie die Bürgermeister-Mehrheit (la majorité mayorale) bzw. unmittelbar den Bürgermeister gestärkt, der sich des Referendums bedient, um seine Entscheidungen beim „Wahlvolk“ direkt abzusichern und dadurch zusätzlich zu legitimieren. ${ }^{22}$ Dabei liegt nicht nur hinsichtlich des Gegenstandbereichs für Referenden, sondern auch was den politik-taktisch bedeutsamen Zeitpunkt der Durchführung betrifft, die Entscheidungsmacht beim

21 Vgl. Paoletti, Pratique politique (Fn. 6), S. 221.

22 Vgl. Rangéon, Levier institutionnel (Fn. 13), S. 55. 
Bürgermeister. ${ }^{23}$ Auch die Entscheidungsergebnisse stützen die Legitimationsfunktion der Referenden zugunsten des Bürgermeisters. So wird - unabhängig von der Größe der Kommune - in der weit überwiegenden Zahl der Fälle die Vorentscheidung des Bürgermeisters bestätigt, und es sind nur sehr seltene Ausnahmefälle (insgesamt sieben) registriert worden, in denen das Abstimmungsergebnis dem Votum des Bürgermeisters entgegenlief. Die Erfolgsquote dieser „Bürgermeisterreferenden“ liegt damit für den Zeitraum 1971 bis 1993 bei 97\%. ${ }^{24}$ Die enge Beschränkung der Referenden auf exekutive Vorstellungen zeigt sich auch darin, dass in nur 20\% aller Referenden überhaupt die Wahl zwischen zwei möglichen Optionen im Hinblick auf ein bestimmtes Vorhaben bestand, wohingegen $80 \%$ der Referenden ein vom Bürgermeister verfochtenes Projekt zum Gegenstand hatten, das durch die (vom Bürgermeister initiierte) Volksabstimmung nur noch zusätzlich bestätigt werden sollte. Anstatt das repräsentativ-demokratische Modell also in Frage zu stellen, wird dieses durch die „Inbesitznahme“ der Referenden von Seiten des Bürgermeisters und seiner Mehrheit gar gestärkt und zementiert.

„Es (das Referendum; S.K.) trägt also um so mehr zur Wiederbelebung und Stärkung des repräsentativen Prinzips bei. ${ }^{25}$

„Seit 1982 haben sicherlich einige kommunale Verantwortliche geglaubt, die Einwohner ihrer Stadt konsultieren zu müssen (...), dies geschah aber eher mit dem Ziel, das persönliche Image aufzupolieren, als um sich der Zustimmung der lokalen öffentlichen Meinung zu vergewissern. ${ }^{26}$

Seit der Einführung bindender Referenden im Jahre 2003 hat sich an dieser Grundtendenz wenig geändert. Als eine Konsequenz der negativen Erfahrungen, die die Zentralregierung mit von ihr initiierten lokalen Referenden in den Überseedepartements machen musste ${ }^{27}$, zeichnet sich vielmehr ab, dass die lokalen

23 Es zeigte sich, dass der strategisch günstigste Zeitpunkt offensichtlich, wie in der Mehrzahl der Fälle festzustellen war, etwa nach Ablauf einer halben Legislaturperiode (mimandat), am häufigsten im dritten oder vierten Jahr, liegt.

24 Siehe Paoletti, Pratique politique (Fn. 6), S. 230.

25 Paoletti, Pratique politique (Fn. 6), S. 231 (Übersetzung S.K.).

26 Mabileau, Kommunalpolitik (Fn. 3), S. 98.

27 Die beiden von der Zentralregierung initiierten, bindenden lokalen Referenden - auf Korsika (6.7.2003) und den Antillen (7.12.2003) - bezogen sich auf die Veränderung des Rechtsstatus der jeweiligen Gebietskörperschaft (collectivité territoriale unique). Sie gingen beide negativ aus. Allerdings gab es auch zwei Referendumserfolge bei der Abstimmung über die Umwandlung der beiden Inseln Saint-Martin und Saint-Barthélémy in eigenständige Übersee-Gebietskörperschaften (siehe L. Lemouzy, Referenda locaux aux DOM-TOM. Deux petits 'Oui' et deux grands 'Non'. In: Pouvoirs Locaux, no. 60 I/2004, 4). 
Mandatsträger auch weiterhin das bindende Referendum meiden und nur dort auf Konsultationen zurückgreifen, wo sie ein für sie vorteilhaftes Ergebnis erwarten können.

„Wenn die Wähler systematisch die Reformvorschläge ablehnen, die ihnen zur Abstimmung vorgelegt werden, wie viele Kommunalpolitiker werden wohl noch riskieren, ein lokales Referendum zu organisieren? “28

Zusammenfassend kann festgehalten werden, dass das lokale Referendum in Frankreich in erster Line ein „Bürgermeister-Referendum“ (,un référendum mayoral $^{(*)}$ ) ist, das dazu beiträgt, diesen und die ihn tragende Ratsmehrheit zu stärken und die lokal-exekutive Macht sogar zu „multiplizieren“ (,un multiplicateur de légitimité pour le maire"). ${ }^{29}$ Somit ist das lokale Referendum in Frankreich auch nicht geeignet, Parteiendifferenzen im Politikergebnis zu überbrücken, zum einen weil diese ohnehin kaum eine Rolle spielen. Zum anderen hat die Abstimmung lediglich die Funktion, eine exekutiv getroffene Politikentscheidung legitimatorisch abzusichern und nicht - wie etwa in Deutschland abzuschwächen oder gar zu kippen.

\section{Stärkung lokaler Macht im Mehrebenensystem}

Das Bürgermeister-Referendum hat sich als ein probates Mittel erwiesen, um lokale Politikvorstellungen gegenüber staatlichen Akteuren zu artikulieren und sich auf übergeordneten Ebenen Gehör zu verschaffen. So bezieht sich etwa ein Drittel der Referenden, die zwischen 1992 und 1999 durchgeführt wurden, auf Sachverhalte, die gar nicht im Kompetenzbereich der Kommunen liegen. Im Zeitraum 1995 bis 2004 lag die Quote der unzulässigen Ratsbegehren (faktisch „Bürgermeisterbegehren“) bei 20\%, was ebenfalls überwiegend darauf zurückzuführen ist, dass über Sachverhalte, die nicht im kommunalen Zuständigkeitsbereich liegen, abgestimmt wurde. Da den repräsentativen Gremien, die die Abstimmungen initiieren, in der Regel die Unzulässigkeit vorher bekannt ist, geht es bei diesen Referenden auch nicht darum, eine Entscheidung in der Sache zu treffen, sondern vielmehr darum, die Position der Kommune hinsichtlich eines bestimmten Entscheidungssachverhalts gegenüber externen (vor allem staatlichen) Akteuren kundzutun. Sie haben oft einen ausgeprägten Charakter als „Protestreferenden“, was umso wirksamer ist, wenn die Bürgermeister, die hinter die-

29 Siehe Paoletti, Pratique politique (Fn. 6), S. 229 f. 
sen Aktionen stehen, zugleich Parlamentsabgeordnete oder Senatoren sind..$^{30}$ Bei diesen „externen“ Protestreferenden, die sich in der Regel einer besonders hohen Beteiligung erfreuen und die teils auch von mehreren kleinen Kommunen gemeinschaftlich organisiert werden, geht es etwa um von staatlichen Stellen (z.B. Präfektur) abgelehnte kommunale Projekte, die öffentlich zu verteidigen dann Gegenstand des Referendums ist. Zum Teil richtet sich die Mobilisierung aber auch gegen Projekte eines Ministeriums im lokalen Raum, oder es handelt sich schlicht um eine Art „formalisierte Massenpetition“ an die Zentralregierung in Paris, wofür das Ausländerwahlrecht für Nicht-EU-Bürger ein Beispiel bietet. Damit eröffnen Referenden einerseits den lokalen Mandatsträgern eine willkommene politische Bühne, um lokale Belange „nach oben“ zu transportieren. Andererseits werden sie genutzt, um nationale Themen über eine lokale Mobilisierung dem politischen Diskurs zu öffnen und die nationalen Entscheidungsträger damit unter Handlungsdruck zu setzen. Sie erfüllen damit eine Artikulationsund Koordinierungsfunktion im Mehrebenensystem und verstärken tendenziell die Ebenenverschränkung und vertikale Politikverflechtung. Den „lokalen Notabeln“ bieten sie einerseits eine weitere Möglichkeit, ihre Macht zu delokalisieren und den Zentralstaat zu „kolonialisieren“. Andererseits eignen sie sich für nationale Repräsentanten als lokale Bühne, um für ihre Politikziele zu werben. Mit den lokalen Referenden haben die Bürgermeister in jedem Falle eine zusätzliche Handlungsoption, um staatliche Akteure „kompromissbereit“ zu stimmen und zur Kooperation zu bewegen, womit sich die verhandlungsdemokratische Ausrichtung im französischen Mehrebenensystem weiter verstärkt.

\section{Einsparungen?}

Ratsinitiierte Referenden haben weder unmittelbar noch mittelbar Einsparungen bewirken können. Abgesehen davon, dass die Anwendungshäufigkeit der Referenden ohnedies begrenzt ist, hat sich gezeigt, dass die Bürgermeister die Referendumsoption häufig nutzen, um Entscheidungen zu kostenträchtigen Projekten, bei denen später auch die Bürger zur Kasse gebeten werden sollen, vorab zu legi-

30 Ein illustratives Beispiel bietet hier die Pariser Vorstadt Saint-Denis, wo der Bürgermeister, der zugleich Parlamentsabgeordneter ist, die Bürger am 26.3.2006 darüber abstimmen ließ, ob sie die Einführung des passiven und aktiven Wahlrechts für Nicht-EU-Ausländer befürworten. Das Referendum war schon zuvor vom zuständigen Verwaltungsgericht für unzulässig erklärt worden. Dennoch stimmten $64 \%$ der Wahlberechtigten zu. Vgl. Le Monde, Les habitants de Saint-Denis favorables au droit de vote des étrangers aux élections locales, Paris 2006, www.lemonde.fr, 26.8.2010. 
timieren und beim Wahlvolk abzusichern. ${ }^{31}$ Allerdings werden die finanziellen Fragen selbst nicht zum Gegenstand des Referendums gemacht, sondern es wird lediglich über das Projekt inhaltlich abgestimmt. So ist es durchaus bezeichnend, dass der für die Kommune zentrale Entscheidungsbereich, der Haushalt, am seltensten zum Gegenstand von Referenden gemacht wird. Seit 1982 haben zudem nur drei Kommunen (Mons-en-Baroeul, Lamotte-Beuvron und Palaiseau) ihre Einwohner über die Anhebung der lokalen Steuern abstimmen lassen. Hierin zeigt sich, dass gerade die wichtige Frage der lokalen Finanzen bewusst vom direkten Bürgervotum ferngehalten wird. Die Anwendung der Referendumsoption durch die Bürgermeister dient eher dazu, zusätzliche Ausgaben, die für ressourcenintensive Politikprojekte mobilisiert werden müssen und damit tendenziell eine Ausweitung des kommunalen Budgets (vor allem durch Steuererhöhungen) erfordern, politisch-legitimatorisch abzusichern.

\section{E. Fazit: Konvergenz oder Divergenz im deutsch-französischen Vergleich?}

Im Vergleich zu Deutschland erweisen sich Frankreichs Reformschritte als sehr gemäßigt. Während hierzulande sowohl das Sachplebiszit als auch das Personalplebiszit flächendeckend eingeführt wurden, beschränkte sich die Diskussion in Frankreich von vornherein auf Abstimmungen zu Sachthemen. Nicht die weitere politisch-institutionelle Aufwertung der lokalen Exekutive, sondern im Gegenteil die Disziplinierung und Begrenzung exzessiver Bürgermeistermacht sind in Frankreich Gegenstand der (allerdings bislang wenig erfolgreichen) Reformbemühungen gewesen. Dagegen richteten sich die Reformmaßnahmen in Deutschland gerade auf die Stärkung von local leadership und effektiver Exekutivmacht, allerdings bei gleichzeitiger Installierung von Gegengewichten in Form des vom Volk initiierbaren bindenden lokalen Referendums.

Auch im Hinblick auf die Institutionalisierung der lokalen Referenden gibt es deutliche Unterschiede im deutsch-französischen Vergleich. Insgesamt stellen die deutschen Reformschritte einen sehr viel konsequenteren Einschnitt in das überkommene repräsentativ-demokratische Modell dar, als dies in Frankreich der Fall ist. Der flächendeckenden Einführung des Bürgerbegehrens in Deutschland (bei gleichzeitigem Ausschluss des Ratsbegehrens in immerhin sechs Flächenländern) steht die ,initiative populaire“ in Frankreich mit ihren restriktiven Anwendungsvoraussetzungen, ihrer thematischen Beschränkung auf Fragen der 
Stadtentwicklung und ihrem ausschließlich konsultativen Charakter gegenüber. Während die Abstimmungsergebnisse von lokalen Referenden in Deutschland grundsätzlich für den Rat bindend sind, waren in Frankreich bis 2003 lediglich „Bürgerkonsultationen“ ohne bindende Wirkung möglich. Auch mit der neuen Gesetzgebung zur Einführung bindender Referenden, die ausschließlich über Ratsbegehren (auf Vorschlag des Bürgermeisters) initiiert und mit prohibitiv wirkenden Verfahrenshürden versehen wurden, ist der französische Gesetzgeber weit hinter den direktdemokratischen Vorstößen der deutschen Bundesländer zurückgeblieben.

Betrachtet man die zur Entscheidung gebrachten Referenden hinsichtlich ihrer Ergebnisse, so ist ein interessanter Unterschied im Ländervergleich darin zu erkennen, dass sich in Deutschland tendenziell Parteiendifferenzen im PolicyOutput nivelliert haben, weil Referenden oft von der Ratsminderheit instrumentalisiert wurden, um Entscheidungen der Ratsmehrheit zu kippen, so dass diese sich dann kompromissbereit verhalten musste, um einen für sie womöglich negativ ausgehenden Bürgerentscheid zu verhindern. Da das lokale Referendum in Frankreich nahezu ausschließlich in der Hand des Bürgermeisters liegt, ist es nicht geeignet, Parteiendifferenzen im Politikergebnis zu überbrücken, zum einen, weil diese ohnehin kaum eine Rolle spielen. Zum anderen hat die Abstimmung lediglich die Funktion, eine exekutiv getroffene Politikentscheidung legitimatorisch abzusichern und nicht - wie in Deutschland - abzuschwächen oder gar zu kippen.

Die Stärkung exekutiver Führerschaft und Präsidialisierung des deutschen Kommunalsystems läuft - unbeschadet der verbleibenden Unterschiede zwischen den Bundesländern - tendenziell auf Annäherung an den französischen Lokalpräsidentialismus hinaus, wenngleich dieser ohne Direktwahl auskommt. Die institutionelle Stärkung des Bürgermeisters gegenüber dem Rat, seine zumindest teilweise zu beobachtende „Emanzipation“ von der Ratsfraktion, sein Machtgewinn gegenüber der Verwaltung und sein präsidialeres Verhalten gegenüber der Bürgerschaft fügen sich in die lokale Variante eines präsidentiellen Regierungssystems ein, wie es der süddeutschen und der französischen Kommunaltradition entspricht. Ähnliche Systemveränderungen hat das französische Modell nicht durchlaufen, sondern dieses ist in seinen Grundmustern durch die Reformen der letzten Jahrzehnte eher bestätigt und sogar weiter bekräftigt worden. Denn es ist nicht gelungen, die exzessive Bürgermeistermacht durch Referenden oder andere lokale Veto-Punkte (Quartiersräte, Konzertierungspflichten etc.) zu zügeln, wohingegen die Referendumsoption (auch wegen ihrer viel weiter reichenden Regelungsform) in Deutschland für die Bürgermeister und Mehrheitsfraktionen ein gefährliches Damoklesschwert ist, das sie zu Kompromissen und 
Kooperationsbereitschaft zwingt. Die französischen Bürgermeister haben sich stattdessen die Beteiligungsinstrumente rasch für ihre eigenen Politikstrategien zu Nutze gemacht, was auch mit den institutionellen Regelungen zusammenhängt, die sie selbst im nationalen Gesetzgebungsprozess entscheidend mitbestimmt haben und die folglich zu einer exekutiven Instrumentalisierung geradezu einladen. Damit laufen die lokalen Demokratiereformen in Deutschland - nimmt man Direktwahl, Referenden und repräsentativ-demokratische Bürgerbeteiligung zusammen - auf effektivere checks and balances im Lokalsystem hinaus, die aber in erster Linie durch Stärkung der Volkssouveränität, nicht aber der parlamentarischen Demokratie (etwa durch eine denkbare Stärkung parlamentarischer Minderheitenrechte) erreicht wurden. Dagegen ist es in Frankreich, obwohl auf die Direktwahl des Bürgermeisters verzichtet worden ist und obgleich das bindende lokale Referendum nunmehr Verfassungsrang besitzt, zur weiteren exekutiven Machthäufung gekommen und eine wirksame Gewaltenteilung im lokalen Politiksystem nicht in Sicht. Referenden stellen in diesem Kontext lediglich ein weiteres probates Instrument exekutiver Machtausübung dar, die den städtischen „Super-Präsidentialismus“ in Frankreich stärkt, während die deutschen Bürgermeister auf Verhandlung und Kompromissbildung angewiesen sind und eine Vielzahl von gewichtigen Vetokräften berücksichtigen müssen. Die formal installierten Machtbeschränkungen im französischen Lokalmodell haben sich in markantem Unterschied zum deutschen Fall entweder als unwirksam und nicht praxisrelevant oder als zusätzliche exekutive Machtressourcen erwiesen.

Im Unterschied zu Deutschland haben die lokalen Referenden in Frankreich eine wichtige Artikulationsfunktion im Mehrebenensystem, da sie oft den Charakter von Protestreferenden und lokal organisierten Massenpetitionen gegen den Zentralstaat annehmen, die den in der nationalen Politik verankerten Bürgermeistern eine zusätzliche politische Bühne bieten. Damit fungiert die direkte Demokratie in Frankreich als ein weiteres Instrument der zentral-lokalen Machtverschränkung, was für den deutschen Fall weitgehend ausgeschlossen ist. Zwar gab es in Ostdeutschland zahlreiche Ratsbegehren, die sich gegen die vom Land beschlossenen Gemeindefusionen richteten (68 von insgesamt 85 Ratsbegehren). ${ }^{32}$ Jedoch ist die lokale Mobilisierung ,nach oben“ im Vergleich zu Frankreich viel weniger wirksam. Sie dringt fast nie auf die Bundesebene vor und basiert unter anderem auf der stärkeren personellen Entkoppelung von über- und untergeordneten Ebenen. 
